\title{
A Novel Template for in-situ Microscopy to Reveal Ferroelectric Switching Mechanisms Across Length Scales
}

\author{
Rohan Dhall ${ }^{1}$, Matthew Cabral ${ }^{1}$, Abinash $\operatorname{Kumar}^{1}$ and James M. LeBeau ${ }^{1}$ \\ ${ }^{1 .}$ Department of Material Science and Engineering, North Carolina State University, Raleigh, NC.
}

When polar materials are subjected to large external electric fields, they often undergo structural changes, which can give them their useful physical properties (such as phase change materials, or ferroelectrics), or at times lead to device failure (such as breakdown). In-situ microscopy offers the ideal approach to study the response of such polar materials to external electric fields. Efforts in this direction are, however, often plagued by challenges of sample preparation. The use of focused ion-beam milling, in particular, tends to degrade device properties, create sample damage, and the unwanted spray of excess Ga can lead to electrical shorts. Furthermore, creating an electron transparent sample, which accurately represents real-world devices is not trivial.

We will discuss our recent development of a novel sample preparation method to overcome these challenges, which incorporates microfabrication techniques with conventional TEM sample preparation (Figure 1). We will show how the approach provides interdigitated comb capacitors over large areas of a high quality TEM specimen. The most important consequence of this scheme, is the high degree of uniformity of the resulting electric field distribution between wired electrodes. This sample geometry allows us to extract representative device characteristics (i.e., Polarization-Voltage curves for ferroelectrics), which are relevant from a technological standpoint (Figure 2).

As an example demonstration, we will discuss results of field-induced changes to the atomic structure and domain orientation in various ferroelectric crystals, including $\mathrm{PMN}-\mathrm{PT}, \mathrm{BaTiO}_{3}, \mathrm{PbTiO}_{3}$, and $\mathrm{KNN}$. A sequence of complementary experimental probes, including polarized optical microscopy, nano-beam diffraction and electron back scatter diffraction, and atomic resolution scanning transmission electron microscopy employed. Studying the same capacitor device with all techniques thus enables correlative microscopy from the micro to atomic length scales. This approach hence provides information previously inaccessible to in-situ microscopy efforts, and opens up the possibility of similar studies of structural changes to materials under externally applied electric fields [3].

\section{References:}

[1] Jablonski, M.L. et al, ACS Applied Materials \& Interfaces 8 (2016).

[2] Nelson, C. T. et al, Science 334 (2011).

[3] This material is based upon work supported by the National Science Foundation, as part of the Center for Dielectrics and Piezoelectrics under Grant Nos. IIP-1361571 and IIP-1361503. The authors acknowledge the use of the Analytical Instrumentation Facility at NCSU, which is supported by the state of North Carolina and the National Science Foundation. M.J.C acknowledges support from the National Science Foundation, as part of NRT-SEAS under Grant No, DGE-1633587 


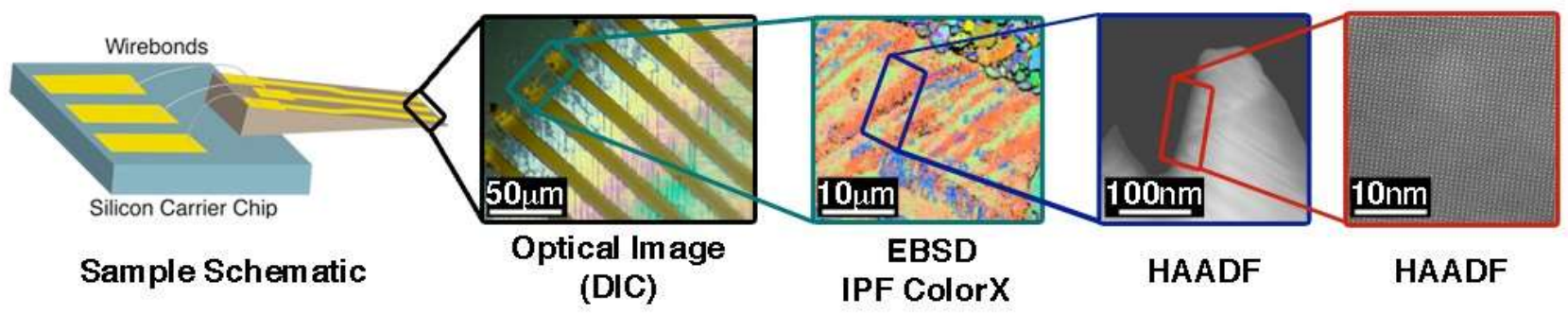

Figure 1. A schematic of electron-transparent interdigitated comb capacitors, compatible with various complementary imaging methods such as polarized optical microscopy, electron backscatter diffraction, and even atomic resolution scanning transmission electron microscopy.
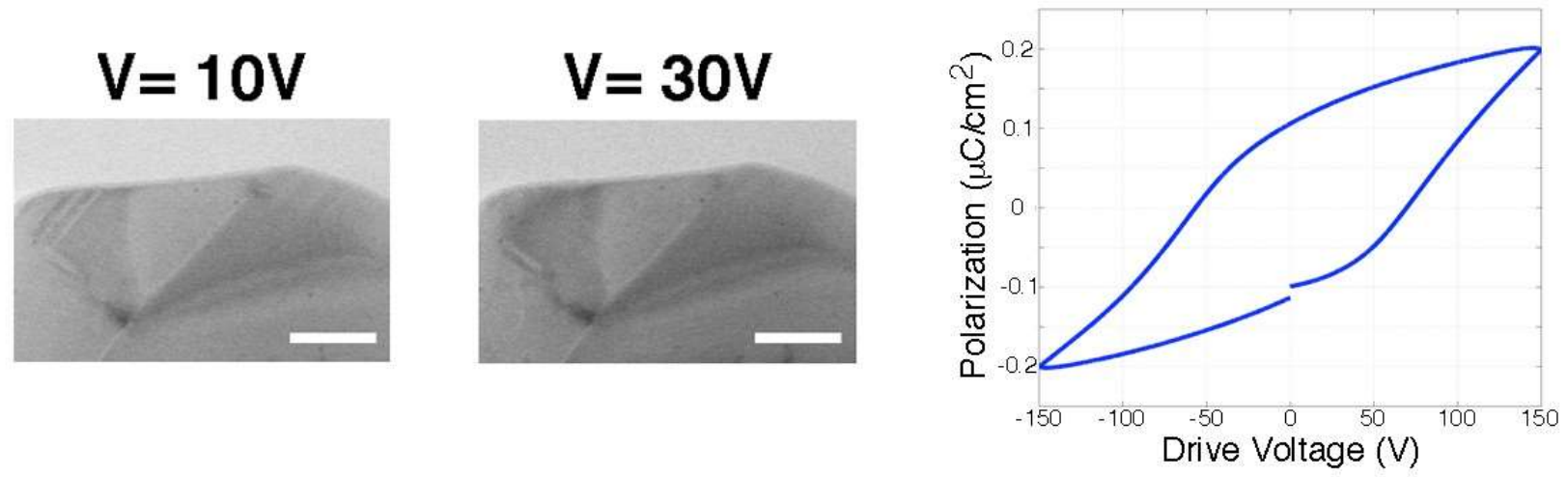

Figure 2. BF-STEM images of $\mathrm{PbTiO}_{3}$ under an applied electric field, showing domain switching (see top left of sample), along with the corresponding hysteresis loop in the polarization-voltage curve. 